\title{
Provision of Remote Psychotherapy during the COVID-19 Pandemic
}

\author{
Elke Humer ${ }^{1} \&$ Thomas Probst ${ }^{2 *}$ \\ 1,2 Department for Psychotherapy and Biopsychosocial Health, Danube University Krems, Krems, Austria \\ elke.humer@donau-uni.ac.at; thomas.probst@donau-uni.ac.at
}

\begin{abstract}
The outbreak of the novel coronavirus disease (COVID-19) creates significant challenges not only for mental health but also for mental health care services. The measures implemented to combat the rapid spread of the virus have created severe barriers to patients accessing in-person, face-to-face psychotherapy services around the world. As direct contact with others should be avoided where possible to prevent virus transmission, adaptations in the therapeutic settings are necessary. Therefore, remote psychotherapy became a valuable option for continuity of mental health care during the COVID-19 pandemic. This article reviews recent studies on the transition to remote psychotherapy during the COVID-19 pandemic. Studies conducted in Austria, the Czech Republic, Germany and Slovakia revealed a strong increase in the provision of psychotherapy via the internet or telephone during the pandemic as compared to the months before. Several differences emerged with regard to country and gender, while the therapeutic orientation was of minor importance. In central Denmark, referrals to psychiatric services declined considerably during the lockdown. But in a psychotherapy program at a public hospital in Massachusetts, a switch to remote psychotherapy led to a reduction in the number of missed appointments. Results imply that the necessary supply of mental health care services could not be sufficiently maintained in all countries during the COVID-19 situation, and that measures are required to further facilitate the provision of mental health care during and after the pandemic.
\end{abstract}

Keywords: psychotherapy, COVID-19, remote psychotherapy, telephone, internet
Article History

Received 9 July 2020

Revised 6 August 2020

Accepted 6 August 2020

DOI 10.24989/dp.v1i2.1868

\section{Introduction}

The novel coronavirus disease-2019 (COVID-19) dramatically impacts global health, economics and social connections around the world (Hasson-Ohayon \& Lysaker, 2020). In an attempt to combat the uncontrolled spread of the virus, measures such as curfews, quarantine, isolation and social distancing have been implemented in many countries (Ghosh et al., 2020). These measures required rapid adaptations in the provision of mental health care around the world (Wind et al., 2020). The protocols implemented to prevent the spread of the virus present a significant challenge to mental health care as face-to-face psychotherapy has to be reduced (Williams \& Tsiligianni, 2020).

Several reviews and meta-analyses highlight that the COVID-19 pandemic and the measures necessary to fight it increase psychological distress and risk of negative mental health consequences in the general population (Brooks et al., 2020; Salari et al., 2020; Vindegaard \& Benros, 2020). These may comprise fears of infection and health anxiety, existential fears, insomnia, depression and post-traumatic stress disorder symptoms (Pfefferbaum \& North, 2020). Especially for individuals with mental health disorders prior to the eruption of the pandemic, a new traumatic or stressful event is likely to aggravate psychological distress and can also trigger prior anxieties and stimulate earlier traumatic memories (Briere \& Scott, 2015; Ronen-Setter \& Cohen, 2020). Therefore, adequate mental health care is of crucial importance in the time of the COVID-19 pandemic.

However, the situation around COVID-19 also poses a challenge for many psychotherapists used to providing treatment through personal contact. To provide mental health care while reducing the risk of transmitting the infection between patients and therapists, in-person, face-to-face psychotherapy is often replaced by remote psychotherapy (Probst et al., 2020; Qiu et al., 2020; Xiang et al., 2020). The provision of mental health care at a safe distance seems to be the obvious solution to ensure sufficient psychotherapeutic support in times of increasing mental health problems (Qiu et al., 2020; Wang et al., 2020; Wind et al., 2020). However, some psychotherapists and their patients have reservations about the use of remote psychotherapy, as the personal contact is often considered as an essential part of the therapy (Apolinário-Hagen et al., 2018; Connolly et al., 2020). If patients and/or therapists are not willing to switch to remote 
psychotherapy, an undersupply of mental health care might emerge. Therefore, this review aims to summarize recent studies on the provision of mental health care during the COVID-19 pandemic.

\section{Studies conducted on changes in the provision of psychotherapy}

During the early weeks of the COVID-19 lockdown in Austria, an online survey was conducted in which all licensed psychotherapists in the list of psychotherapists of the Austrian Federal Ministry of Social Affairs, Health, Care and Consumer Protection were contacted (all those for which a valid email address was available). In total 1,547 psychotherapists participated in the survey, which revealed that the total number of patients treated on average per week in the early weeks of the COVID-19 lockdown was on average $28 \%$ lower compared to the months before the lockdown $(p<.001)$ (Probst et al., 2020). Besides this change in the total number of patients treated per week, there was also a significant change in the mode of delivery. While on average most patients were treated through personal contact before the COVID-19 pandemic, the most preferred treatment format in the early weeks of the lockdown was telephone, followed by internet. In more detail, the number of patients treated per week via personal contact decreased by on average $81 \%$ $(p<.001)$ during the COVID-19 lockdown, while the number of patients treated on average per week via telephone increased by on average $979 \%(p<.001)$. The strongest relative change was observed for the number of patients treated on average per week via the internet, which increased on average by $1,561 \%$ during the COVID-19 lockdown as compared to the months before $(p<.001)$. However, the increase in remote psychotherapy (telephone + internet) was not sufficient to compensate for the strong decrease in the provision of in-person, face-to-face treatment $(p<.001)$, indicating an undersupply of mental health care during the early weeks of the COVID-19 lockdown in Austria. In addition, possible differences among therapeutic orientations (behavioral, humanistic, psychodynamic, and systemic) were investigated, showing comparable changes between the four therapeutic orientations in the changes in the provision of psychotherapy.

An online survey of 112 psychotherapists in the Czech Republic was conducted two weeks after lockdown measures were lifted, in which participants were contacted via email using the list of the Czech Association for Psychotherapy. The study revealed that the total number of patients treated on average per week in the months before the COVID-19 remained the same as the number of patients treated during the COVID-19 pandemic ( $p=.086$ ) (Humer et al., 2020). However, significant changes in the way psychotherapy was provided were observed, with a $71 \%$ decrease in the number of patients treated on average per week via personal contact $(p<.001)$. The number of patients treated via telephone increased by on average $417 \%$ $(p<.001)$. The strongest change was observed for the number of patients treated via the internet, which increased by on average $1,200 \%$ during the COVID-19 situation $(p<.001)$. Overall, the preferred treatment format before the COVID-19 pandemic of face-to-face psychotherapy shifted to psychotherapy via the internet during the COVID-19 pandemic. However, more patients were still treated in personal contact as compared to telephone during the COVID-19 pandemic.

In Germany, an online survey of psychotherapists was conducted two weeks after the lockdown measures began to be lifted. Participants were contacted via e-mail, whereby the addresses were gathered from the directories of four different regional and national psychotherapeutic associations. In total, 130 therapists participated in the survey, which revealed that the number of patients treated on average per week actually increased by on average $12 \%(p=.014)$ during the COVID-19 situation as compared to the months before (Humer et al., 2020). Although the number of patients treated on average per week in personal contact decreased by $18 \%(p<.001)$, face-to-face psychotherapy remained the most common treatment format during the COVID-19 pandemic. Significant increases in remote psychotherapy were also observed, with an average increase in the number of patients treated via telephone on average per week of $213 \%$, $(p=.001)$, and via internet of $6,558 \%(p<.001)$.

Three weeks after lockdown restrictions began to be lifted in Slovakia, psychotherapists were asked about the number of patients treated on average per week in the months before the COVID-19 pandemic as compared to the current situation (Humer et al., 2020). The chair of the Slovak Psychotherapeutic Society and the chairs of special psychotherapeutic societies invited the psychotherapists to provide input via email. In total 96 psychotherapists participated. A decrease in the total number of patients treated per week by on average $25 \%(p<.001)$ was observed. Before the pandemic most patients were treated faceto-face. This altered considerably during the COVID-19 situation, with an average decrease of the number of patients treated face-to-face per week by $76 \%(p<.001)$. This decrease could not be compensated for by increases in remote psychotherapy, although the number of patients treated on average per week via telephone increased on average by $187 \%(p<.001)$, and the number of patients treated on average per week via the internet increased on average by $343 \%(p<.001)$.

Among the surveys conducted in the Czech Republic, Germany and Slovakia a stronger reduction of in-person, face-toface psychotherapy was observed for female psychotherapists, showing a lower number of patients treated via personal contact $(p=.036)$ and a higher number of patients treated via telephone ( $p=.015)$ during the COVID-19 pandemic as compared to the months before. In the months before the COVID-19 situation, no differences between male and female psychotherapists were observed with regard to the mode through which psychotherapy was provided ( $p \geq 0.725$ ) (Humer et al., 2020).

Different explanations exist for the varying changes in the provision of psychotherapy among countries. First, there are 
country-specific regulations regarding the provision of psychotherapy via the internet or telephone. For instance, in Austria internet-based psychotherapy was rejected by the Austrian Internet guideline for psychotherapists (Bundesministerium für Gesundheit und Frauen, 2005). Second, there are differences between countries in relation to the reimbursement of the costs of psychotherapy via the internet by insurance companies. Third, different time points (during the lockdown in Austria, after the lockdown in the Czech Republic, Germany and Slovakia) have to be considered as legal restrictions, regulations of insurance companies regarding the reimbursement of costs of remote psychotherapy, preferences in the format psychotherapy is provided as well as risks and fears of COVID-19 infections likely change dynamically. Fourth, there are also likely differences between countries in relation to the fear of infection due to participation in face-to-face psychotherapies, and also with respect to gender. Indeed, the lowest fear of infection was observed in German psychotherapists as well as male psychotherapists, a group which showed the smallest reduction in the provision of face-toface psychotherapy (Humer et al., 2020). Fifth, experience in the use of digital media might also differ among countries.

\section{Studies conducted on changes in referrals to psychiatric services}

In Denmark all referrals to psychiatric services in the first weeks of the nationwide curfew were compared to the corresponding weeks in the previous year (Kølbæk et al., 2020). A total reduction of $40 \%$ was observed. This is especially concerning, as in the weeks preceding the lockdown in 2020, almost $20 \%$ more referrals were observed as compared to the corresponding weeks in 2019. During the weeks of gradual lifting of the lockdown in 2020 , the number of referrals was almost comparable to those of the corresponding weeks in 2019 (on average -6\%). As it is unlikely that the mental health improved during the COVID-19 lockdown, it seems more reasonable that patients with mental health issues were not referred to psychiatric services, possibly due to the fear of this leading to an increase in COVID-19 infections.

A recent report on mental health care in the USA and Israel focusing on individuals with schizophrenia highlights the initial difficulties faced by many patients with the shift from face-toface psychotherapy to psychotherapy via telephone or videoconferencing, such as limited access to the required technology and reduced privacy (Hasson-Ohayon \& Lysaker, 2020). However, positive aspects were also reported by some patients, such as an ease to talk openly when not being present in the same room as the therapist, which was highlighted particularly when psychotherapy was conducted using the telephone.

A study investigating the caseloads in an outpatient psychodynamic psychotherapy training program at a public hospital in Cambridge (Massachusetts) revealed a substantial decrease in missed appointments upon switching to remote mental health services (Silver et al., 2020). While the mean missed appointment rate before the transition to remote psychotherapy was approximately $14 \%$, it decreased to approximately $6 \%$ $(p=0.03)$ after the transition to remote psychotherapy. Possible explanations reported by the authors are: a higher need for human relationships and contact during crises; a higher ability to tolerate attending sessions due to an additional layer of insulation between patient and psychotherapists in psychotherapy conducted from a distance; and a reduction of barriers to treatment, such as logistical or family-related challenges.

\section{Technical-based applications}

There are a number of technical-based e-mental health applications that have the potential to extend treatment far beyond the provision of psychotherapy via the internet or telephone in the current crisis. Several guided but also self-guided interventions, such as self-help apps or online therapeutic modules, might also be a valuable option in the provision of mental health care (Wind et al., 2020), especially in countries with scare mental health resources (Christiani and Setiawan, 2018). E-mental health applications have already been developed for a wide range of psychiatric conditions. These are cost-effective and have displayed promising outcomes, in some cases being shown to be as effective as face-to-face psychotherapy (Andersson, 2016, 2018). However, there are also several limitations of replacing psychotherapy with technical-based e-mental health applications. One is that psychiatric diagnosis cannot reliabely made solely using self-reports, as unless the patient is seen by a mental health care professional, important information may be lost. A further drawback is the difficulty of validating the accuracy of responses and of obtaining additional information. Studies report higher drop-out rates from internet interventions as compared to face-to-face interventions, and indicate improved adherence to a treatment program with the involvement of therapist support. A further important aspect is that the highprevalence of comorbidity is to date not adequately addressed by e-mental health applications, as these applications commonly target specific disorders. Finally, issues related to user privacy have to be considered, which is relevant for data collection and storage, especially when smartphone applications are used (Andersson \& Titov, 2014; Cunningham et al., 2014). Therefore, future studies are needed to assess the potential of e-mental health applications to replace psychotherapy, both during and after the COVID-19 pandemic.

\section{Conclusions}

To summarize, although efforts to substitute face-to-face psychotherapy with psychotherapy at a safe distance have been made in many countries, these attempts have not been sufficient to provide adequate mental health care services in all investi- 
gated countries. In general, studies reported that the actual effectiveness of the psychotherapy, treatment satisfaction and the therapeutic alliance can be achieved equally by psychotherapy via telephone or the internet as in face-to-face psychotherapies (Backhaus et al., 2012; Bashshur et al., 2016; Jenkins-Guarnieri et al., 2015; Langarizadeh et al., 2017; Lopez et al., 2019). Therefore, it is important to highlight the utility of remote psychotherapy to those psychotherapist and patients who have concerns to maintain mental health care in times of COVID-19. Overall, this review implies that remote psychotherapy initiatives are required in mental health care systems in several countries to cover the need for professional mental health care during and after the COVID-19 pandemic.

\section{References}

Andersson, G. (2016). Internet-delivered psychological treatments. Annual Review of Clinical Psychology, 12(1), 157-179. https://doi. org/10.1146/annurev-clinpsy-021815-093006

Andersson, G. (2018). Internet interventions: Past, present and future. Internet Interventions, 12, 181-188. https://doi.org/10.1016/j.invent. 2018.03.008

Andersson, G., \& Titov, N. (2014). Advantages and limitations of Internet-based interventions for common mental disorders. World Psychiatry, 13(1), 4-11. https://doi.org/10.1002/wps.20083

Apolinário-Hagen, J., Harrer, M., Kählke, F., Fritsche, L., Salewski, C., \& Ebert, D. D. (2018). Public attitudes toward guided internetbased therapies: Web-based survey study. JMIR Mental Health, 5(2), e10735. https://doi.org/10.2196/10735

Backhaus, A., Agha, Z., Maglione, M. L., Repp, A., Ross, B., Zuest, D., Rice-Thorp, N. M., Lohr, J., \& Thorp, S. R. (2012). Videoconferencing psychotherapy: A systematic review. Psychological Services, 9(2), 111-131. https://doi.org/10.1037/a0027924

Bashshur, R. L., Shannon, G. W., Bashshur, N., \& Yellowlees, P. M. (2016). The empirical evidence for telemedicine interventions in mental disorders. Telemedicine and E-Health, 22(2), 87-113. https:// doi.org/10.1089/tmj.2015.0206

Briere, J., \& Scott, C. (2015). Complex trauma in adolescents and adults. Psychiatric Clinics of North America, 38(3), 515-527. https://doi. org/10.1016/j.psc.2015.05.004

Brooks, S. K., Webster, R. K., Smith, L. E., Woodland, L., Wessely, S., Greenberg, N., \& Rubin, G. J. (2020). The psychological impact of quarantine and how to reduce it: Rapid review of the evidence. The Lancet, 395(10227), 912-920. https://doi.org/10.1016/S01406736(20)30460-8

Bundesministerium für Gesundheit und Frauen. (2005). Internetrichtlinie für Psychotherapeutinnen und Psychotherapeuten. 13, 43-51.

Connolly, S. L., Miller, C. J., Lindsay, J. A., \& Bauer, M. S. (2020). A systematic review of providers' attitudes toward telemental health via videoconferencing. Clinical Psychology: Science and Practice. https:// doi.org/10.1111/cpsp.12311

Cunningham, J. A., Gulliver, A., Farrer, L., Bennett, K., \& CarronArthur, B. (2014). Internet interventions for mental health and addictions: Current findings and future directions. Current Psychiatry Reports, 16(12), 521. https://doi.org/10.1007/s11920-014-0521-5
Ghosh, A., Mukherjee, K., Dasgupta Ghosh, B., \& Dutta, S. (2020). COVID-19 outbreak - The role of worldwide interventions as a preventive measure to fight the pandemic. SSRN Electronic Journal. https://doi.org/10.2139/ssrn.3599808

Hasson-Ohayon, I., \& Lysaker, P. H. (2020). Special challenges in psychotherapy continuation and adaption for persons with schizophrenia in the age of coronavirus (COVID-19). Counselling Psychology Quarterly, 1-9. https://doi.org/10.1080/09515070.2020.1781595

Humer, E., Pieh, C., Kuska, M., Barke, A., Doering, B. K., Gossmann, K., Trnka, R., Meier, Z., Kascakova, N., Tavel, P., \& Probst, T. (2020). Provision of psychotherapy during the COVID-19 pandemic among Czech, German and Slovak psychotherapists. International Journal of Environmental Research and Public Health, 17(13), 4811. https:// doi.org/10.3390/ijerph17134811

Jenkins-Guarnieri, M. A., Pruitt, L. D., Luxton, D. D., \& Johnson, K. (2015). Patient perceptions of telemental health: Systematic review of direct comparisons to in-person psychotherapeutic treatments. Telemedicine and E-Health, 21(8), 652-660. https://doi.org/10.1089/ tmj.2014.0165

Kølbæk, P., Nørremark, B., \& Østergaard, S. D. (2020). Forty percent reduction in referrals to psychiatric services during the COVID-19 pandemic. Psychotherapy and Psychosomatics, 1-2. https://doi.org/ 10.1159/000509575

Langarizadeh, M., Tabatabaei, M., Tavakol, K., Naghipour, M., \& Moghbeli, F. (2017). Telemental health care, an effective alternative to conventional mental care: A systematic review. Acta Informatica Medica, 25(4), 240. https://doi.org/10.5455/aim.2017.25.240-246

Lopez, A., Schwenk, S., Schneck, C. D., Griffin, R. J., \& Mishkind, M. C. (2019). Technology-based mental health treatment and the impact on the therapeutic alliance. Current Psychiatry Reports, 21(8), 76. https://doi.org/10.1007/s11920-019-1055-7

Pfefferbaum, B., \& North, C. S. (2020). Mental health and the Covid-19 pandemic. New England Journal of Medicine, NEJMp2008017. https://doi.org/10.1056/NEJMp2008017

Probst, T., Stippl, P., \& Pieh, C. (2020). Changes in provision of psychotherapy in the early weeks of the COVID-19 lockdown in Austria. International Journal of Environmental Research and Public Health, 17(11), 3815. https://doi.org/10.3390/ijerph17113815

Qiu, J., Shen, B., Zhao, M., Wang, Z., Xie, B., \& Xu, Y. (2020). A nationwide survey of psychological distress among Chinese people in the COVID-19 epidemic: Implications and policy recommendations. General Psychiatry, 33(2), e100213. https://doi.org/10.1136/ gpsych-2020-100213

Ronen-Setter, I. H., \& Cohen, E. (2020). Becoming "teletherapeutic": Harnessing Acelerated Experiential Dynamic Psychotherapy (AEDP) for challenges of the Covid-19 era. Journal of Contemporary Psychotherapy. https://doi.org/10.1007/s10879-020-09462-8

Salari, N., Hosseinian-Far, A., Jalali, R., Vaisi-Raygani, A., Rasoulpoor, S., Mohammadi, M., Rasoulpoor, S., \& Khaledi-Paveh, B. (2020). Prevalence of stress, anxiety, depression among the general population during the COVID-19 pandemic: A systematic review and meta-analysis. Globalization and Health, 16(1), 57. https://doi.org/ 10.1186/s12992-020-00589-w

Silver, Z., Coger, M., Barr, S., \& Drill, R. (2020). Psychotherapy at a public hospital in the time of COVID-19: Telehealth and implications for practice. Counselling Psychology Quarterly, 1-9. https://doi.org/1 0.1080/09515070.2020.1777390 
Thompson-de Benoit, A., \& Kramer, U. (2020). Work with emotions in remote psychotherapy in the time of Covid-19: A clinical experience. Counselling Psychology Quarterly, 1-9. https://doi.org/10.108 0/09515070.2020.1770696

Vindegaard, N., \& Benros, M. E. (2020). COVID-19 pandemic and mental health consequences: Systematic review of the current evidence. Brain, Behavior, and Immunity, S0889159120309545. https:// doi.org/10.1016/j.bbi.2020.05.048

Wang, C., Pan, R., Wan, X., Tan, Y., Xu, L., Ho, C. S., \& Ho, R. C. (2020). Immediate psychological responses and associated factors during the initial stage of the 2019 coronavirus disease (COVID-19) epidemic among the general population in China. International Journal of Environmental Research and Public Health, 17(5), 1729. https:// doi.org/10.3390/ijerph17051729

Williams, S., \& Tsiligianni, I. (2020). COVID-19 poses novel challenges for global primary care. Npj Primary Care Respiratory Medicine, 30(1), 30, s41533-020-0187-x. https://doi.org/10.1038/s41533-020$0187-\mathrm{x}$

Wind, T. R., Rijkeboer, M., Andersson, G., \& Riper, H. (2020). The COVID-19 pandemic: The 'black swan' for mental health care and a turning point for e-health. Internet Interventions, 20, 100317. https:// doi.org/10.1016/j.invent.2020.100317

Xiang, Y.-T., Yang, Y., Li, W., Zhang, L., Zhang, Q., Cheung, T., \& Ng, C. H. (2020). Timely mental health care for the 2019 novel coronavirus outbreak is urgently needed. The Lancet Psychiatry, 7(3), 228-229. https://doi.org/10.1016/S2215-0366(20)30046-8

\section{${ }^{*}$ Corresponding Author}

\section{Thomas Probst}

Department for Psychotherapy and Biopsychosocial Health Danube University Krems

Dr.-Karl-Dorrek-Straße 30

Krems, 3500, Austria

Tel: +43 2732893-2679

E-mail: Thomas.Probst@donau-uni.ac.at

\section{Declaration of Interest}

All authors declare no conflict of interest.

\section{Funding}

No funding. 